\title{
Variations on a Theme: Crispr Models for 15q11-q13 Disorders and Beyond
}

\section{Barbara J Bailus}

Buck Institute for Research on Aging, Novato, CA 94945, USA

*Corresponding author: Barbara J. Bailus, Buck Institute for Research on Aging, Novato, CA 94945, USA, Tel: 1-707-235-6514; E-mail: bjbailus@ucdavis.edu

Rec date: Aug 14, 2014, Acc date: Sep 06, 2014, Pub date: Sep 25, 2014

Copyright: (C) 2014 Bailus BJ. This is an open-access article distributed under the terms of the Creative Commons Attribution License, which permits unrestricted use, distribution, and reproduction in any medium, provided the original author and source are credited.

\begin{abstract}
Objective: Genetic disorders involving the 15q11-q13 region in humans are complex and often exhibit a variety of mutation types that can affect multiple genes in the region. Angelman syndrome and Prader-Willi syndrome are neurodevelopmental disorders that are caused by mutations in this region. A variety of mutations, from point to large deletions are exhibited in the patient population. Although the mutations that cause several of these disorders are well understood and characterized the availability of in-vivo models that accurately reflect the high variation of mutations in individual patients has not been feasible. This review examines how applying CRISPR technology can result in more accurate models of 15q11-q13 disorders and other multiple gene disorders, including autism spectrum disorders.
\end{abstract}

Background: Previous methods of creating disease models have been cost and labor intensive making it impossible to accurately represent the variation in complex genetic disorders. The advancement of CRISPR technology has drastically changed the ease of producing in-vivo models for diseases where the mutation varies in individuals.

Methods: A review of relevant literature on Angelman syndrome, Prader-Willi syndrome, and CRISPR technology, and the implications of applying CRISPR technology to the autism field.

Results: CRISPR technology has the potential to drastically impact the 15q11-q13 disorders and autism field in the creation of more varied and accurate in-vivo models, which will advance our understanding of these diseases and potentially lead to better treatments.

Keywords: CRISPR; Prader-Willi syndrome; Angelman syndrome; Autism spectrum disorders; 15q11-q13; iPSC

\section{Abbrevations:}

CRISPR: Clustered Regularly Interspaced Short Palindromic Repeat; TALE: Transcription Activator-Like Effector; ZF: Zinc Finger; AS: Angelman Syndrome; PWS: Prader-Willi syndrome; ASD: Autism Spectrum Disorder

\section{Introduction}

Disorders of the 15q11-q13 region have been difficult to model as there exists a wide variation in mutation types that have caused these disorders. Although the minimal mutation region is known for both Angelman Syndrome (AS) and Prader-Willi syndrome (PWS) few patients have these microdeletions, instead patients have a variety of mutations including point mutations, large deletions of multiple genes, imprint center control mutations and uniparental disomy $[1,2]$. This variation of mutations has been difficult to replicate in both animal and cellular models, instead there has been a heavy reliance on one predominant model being used for a majority of the studies $[3,4]$. A similar situation of multiple models being necessary for a disorder can be found in autism spectrum disorders (ASD), a percentage of individuals with 15q11-q13 disorders are also classified on the autism spectrum. Over 100 candidate genes are implicated in ASD, of which a combination of these genes may be mutated in a single individual [5].
Until recently the task of making multiple disease models with varied and exact deletion sizes or multiple gene mutations was extremely labor and cost intensive. The advent of CRISPR (clustered regularly interspaced short palindromic repeats) has the ability to dramatically reduce both time and cost in creating a vast variety of mutation sizes and multiple gene mutations [6]. This review will focus on the advantages the CRISPR technology offers in creating more varied and accurate models for 15q11-q13 associated conditions of Angelman Syndrome and Prader-Willi syndrome and the ability of the technology to be applied beyond into the ASD field, or any genetic disease.

\section{Background}

Angelman Syndrome and Prader-Willi syndrome are neurodevelopmental disorders of which a subpopulation are often placed on the autism spectrum [5,7-9]. Angelman syndrome affects 1:10,000 individuals and symptoms include ataxia, intellectual disability, sleep disorders and in some cases seizures $[2,10,11]$. PraderWilli syndrome affects

1:10,000 individuals and symptoms include obesity, intellectual impairment, sleep disorders and hyperphagia $[2,12]$. Both diseases have been shown to occur with mutations ranging from a single point mutation to large gene spanning deletions of $4 \mathrm{MB}$, making the application of a single model representative of each individual case insufficient $[1,13]$. The causative gene in Angelman syndrome is $U B E 3 A$, a ubiquitin ligase protein that adds ubiquitin as a post- 
translational modification to target proteins for degradation $[14,15]$ In Prader-Willi syndrome the causative mutation is in the SNORD 116 cluster, a group of small nucleolar RNAs that help with protein folding $[12,16,17]$. Cellular iPSC models have been derived from a small number of AS and PWS patients, but represent only a fraction of the mutation variability in the patient population [18]. Patient derived cells can be an excellent source of information, but the genetic background of each patient is different making it difficult to study the exact changes that occur based only on a combination of mutations or the size of a specific deletion. Ideal cellular models would have an identical genetic background and only a mutation variance. The introduction of induced pluripotent stem cells (iPSCs) has made it possible to create many cellular types with an identical genetic background, in an effort to understand the effect of the mutations across various cell types [19-21]. The ability to fully utilize isogenic iPSC models was limited until recently by the time consuming methods with which to introduce new mutations into an iPSC line [22, 23].

The mouse models of Angelman and Prader-Willi disease focus on recapitulating the disease phenotypes, sometimes at the expense of an accurate mutation representation. The most well characterized Angelman syndrome mouse model has a deletion in the $\mathrm{N}$ - terminal section of the protein, effectively deleting many of the known isoforms of Ube3a [3]. This model does not reflect on a genetic level the large deletions seen in $70 \%$ of the patients, the point mutations or imprinting control region mutations seen in another $20 \%$ of the patients [13]. In many Angelman patients multiple genes are deleted in this region, attempts to mimic this large deletion have not been very successful, resulting in poor viability or inexact targeting with traditional homologous recombination technology [24-26]. PraderWilli syndrome models have also been similarly limited, with several of the models only having a portion of the large deletion, though this had been due in part to extremely early mortality in large deletion models [4,26]. All of the current models were time and cost consuming to create and are limited in their ability to represent the full spectrum of variation of mutations in actual patients. This limitation has been problematic for both understanding the disease mechanism and range of symptoms and developing effective treatments. CRISPR technology can help with establishing new and more varied models of 15q11-q13 disorders and the same technology could be applied to ASD.

CRISPR is the latest generation in the genome engineering tools that have been discovered and characterized in the last twenty years [27]. CRISPR technology is a gene editing nuclease system, found in many bacteria and archea [28]. The most well studied of the CRISPR nucleases is Cas9 which is targeted to a specific site in the genome by a guide RNA (gRNA) [6] The guide RNA (gRNA), specifies 18-21bp in the DNA, making it possible to target a single unique site in the genome [29]. Unlike the previous genome engineering tools, zinc fingers and TALENs (transcription activator-like effector nucleases), CRISPR does not require any specialized expertise to use, targets any site in the genome with high efficiency, can be made to order, and has minimal off target effects [30]. Recent studies have shown that the offtarget effects of CRISPR are minimal with as little as one off target site that was cleaved [31,32]. This low off-target cut rate is especially important when introducing a mutation for a disease model, to ensure that only one mutation is introduced into the genome. CRISPR has been used to successfully make several iPSC disease models and mammalian models including the generation of a mouse model with multiple specific mutations in a single generation [33-36]. Zinc fingers exhibit a significant rate of off-target binding, making them less ideal tools for disease model creation [37-39]. TALENS appear to be more specific then ZFN, however they are complicated to create and unlike the CRISPR system two TALENs are needed per desired mutation site, requiring several plasmids/viral vectors for a multiple gene disorder model [39-43]. CRISPR offers three distinct advantages when compared to previous genome engineering tools, high accuracy, ease of design and construction, the ability to fit multiple gRNAs on the plasmid/viral vector with the Cas9 nuclease drastically increasing the efficiency of creating a multiple gene disorder as only one plasmid/ vector is required for the cell or embryo [44,45].

\section{Discussion}

An ideal disease model has the gene causing mutation and no other background mutations, in this way the disease phenotype observed is due only to the single gene mutation. The previous generation of such models was very low efficiency and often utilized a selectable marker, adding an artificial element to the disease model, and potentially even the protein $[22,46]$. Using patient derived cell lines would allow for a representation of the variety of mutations, but there would be large amount of background noise across the various models as each individual patient's genome is different with potentially other mutations in different genes. For a cellular model iPSCs are the ideal model in which to do a series of deletions of varying lengths and a variety of mutations reflective of the patient population, minus the genetic background noise of the population. Using iPSCs, isogenic lines could be established from a wild-type individual so that in the disease model iPSC derived tissues the only variance would be mutations and the size of the mutation if applicable. An additional benefit to utilizing iPSCs is their ability to be reprogramed into any cell type, allowing for observation of the disease phenotype across many cell types, which all share an identical genome. The CRISPR system has been used with high efficiency and accuracy to generate many iPSC cell models, including other neurological diseases $[35,47,48]$. The creation of several models of various $15 \mathrm{q} 11-\mathrm{q} 13$ or ASD mutations would be a simple extension of the current technology. Large deletions could be done by having two gRNAs, which would target appropriately spaced genetic sites, creating large deletions [49]. To reflect multiple gene disorders, the Cas9 nuclease can be used with multiple gRNAs, to create a model in a single step experiment. The CRISPR system has been shown to be highly efficient and accurate, with minimal off target effects and induced mutation rates as high as $15 \%[32,50]$. The created models could be screened for off-target events by a whole genome sequence, which can be done in a time efficient and cost effective manner, ensuring that the model has only one insertion event $[31,32,51]$.

The CRISPR system has been used to efficiently generate a wide variety of disease models in different model organisms, including primates by inserting the Cas9 DNA, gRNA and donor DNA in the embryo [33,52,53]. The 15q11-q13 region disorders and autism spectrum diseases could benefit from this methodology as several forms of autism are caused by multiple mutations in different genes [5,54-56]. Occasionally, in humans the deletion region encompasses genes that are not found in the same region on the mouse chromosome. Until CRISPR, the concept of creating mutations across multiple sites in the genome, on different chromosomes required many generations of mice and different founder lines. The CRISPR system allows for the generation of multiple gene mutations in a single generation, by designing and injecting the multiple gRNAs into the 
desired embryo. Alternatively, if deletion of such a large region proved lethal or unstable then multiple mutations could be made in the genes of interest simultaneously, creating a model in a single generation $[26,33]$. These models would also help elucidate the interplay between multiple gene mutations that cause autism. This technology makes the establishment of these varied models efficient and achievable in any laboratory. Continued modifications of the CRISPR system have reduced off-target mutations to almost background levels, ensuring the model phenotypes accurately reflect the molecular underpinnings of the desired disease [51,57-60].

In an effort to streamline the screening of viable disease treatments, cell lines with specifically tagged proteins could be made with the CRISPR system. These lines could be utilized in the same way the current Ube3a-YFP lines have been utilized, as a high throughput screening method for small molecule drugs that activate paternal Ube3a, in an effort to find therapeutics for Angelman syndrome [46,61]. This use of Ube3a-YFP was pivotal in identifying a class of drugs, topoisomerases, which activated paternal Ube3a [61,62]. The activation of paternal Ube3a lead to a better understanding of how paternal Ube3a was silenced and if activation of paternal Ube3a could compensate for screening efficiency and could even help elucidate protein interactions. Before the optimization of CRISPR technology the tagging of over 100 ASD candidate genes would have been a very time consuming and labor intensive task, now it is a goal that could be accomplished by a single laboratory [5]. A study done on a much larger scale did a whole genome CRISPR knockout screening to identify genes involved in cancer, this study targeted 18,080 genes with 64,751 gRNAs [63]. The scale on which this was done has made the actual targeting of the gene not a limiting factor, the limiting factor in the model creation could be the selecting of the cell clones, which would be enhanced by the insertion of a selectable marker. The creation of an entire library of isogenic iPSCs for 15q11-q13 mutations and every ASD candidate gene is a realistic goal, with the optimization of CRISPR technology. An establishment of this library will make massive parallel drug screening possible, making the screening of potential therapies rapid and straightforward.

\section{Conclusion}

Increased availability of varying deletion length models or multiple gene mutation models will dramatically impact both the basic understanding of 15q11-q13 and autism spectrum disorders, enhancing the potential to find effective treatments for the diseases. Increased availability of varied deletion size and multiple gene mutation disease models with minimal genetic background noise in isogenic iPSCs, tissues and animal models will increase understanding of the underlying molecular mechanism, which may expand options of potential therapies. It is possible that the deletion size, or having multiple gene mutations could impact drug response and efficacy, something that could be tested in created models. This strategy is by no means an all-inclusive model, but the implementation of CRISPR technology would allow for a more representative set of models to advance the molecular and behavioral understanding of 15q11-q13 disorders and ASD, while increasing the ability to evaluate the efficacy of potential therapies.

\section{Acknowledgements}

The author would like to thank Henriette O'Geen for her careful reading of the manuscript, Lisa Ellerby, David J Segal and Mahru An for insightful conversations. BJB was supported by an NSF graduate fellowship (0707429) and a grant to UC Davis from the Howard Hughes Medical Institute through the Med into Grad Initiative (56005706).

\section{References}

1. Chamberlain SJ, Lalande M (2010) Neurodevelopmental disorders involving genomic imprinting at human chromosome 15q11-q13. Neurobiol Dis 39: 13-20.

2. Horsthemke B, Wagstaff $\mathrm{J}$ (2008) Mechanisms of imprinting of the Prader-Willi/Angelman region. Am J Med Genet A 146A: 2041-2052.

3. Jiang YH, Armstrong D, Albrecht U, Atkins CM, Noebels JL et al. (1998) Mutation of the Angelman ubiquitin ligase in mice causes increased cytoplasmic p53 and deficits of contextual learning and long-term potentiation. Neuron 21: 799-811.

4. Bervini S, Herzog H (2013) Mouse models of Prader-Willi Syndrome: a systematic review. Front Neuroendocrinol 34: 107-119.

5. Betancur C (2011) Etiological heterogeneity in autism spectrum disorders: more than 100 genetic and genomic disorders and still counting. Brain Res 1380: 42-77.

6. Jinek M, Chylinski K, Fonfara I, Hauer M, Doudna JA, et al. (2012) A programmable dual-RNA-guided DNA endonuclease in adaptive bacterial immunity. Science 337: 816-821.

7. Curran S, Roberts S, Thomas S, Veltman M, Browne J, et al. (2005) An association analysis of microsatellite markers across the Prader-Willi/ Angelman critical region on chromosome 15 (q11-13) and autism spectrum disorder. Am J Med Genet B Neuropsychiatr Genet 137B: 25-28.

8. Veltman MW, Craig EE, Bolton PF (2005) Autism spectrum disorders in Prader-Willi and Angelman syndromes: a systematic review. Psychiatr Genet 15: 243-254.

9. Rangasamy S, D'Mello SR, Narayanan V (2013) Epigenetics, autism spectrum, and neurodevelopmental disorders. Neurotherapeutics 10: 742-756.

10. Chamberlain SJ, Lalande M (2010) Angelman syndrome, a genomic imprinting disorder of the brain. J Neurosci 30: 9958-9963.

11. Angelman H (1965) Puppet Children a Report on Three Cases. Develop. Med. Child Neurol

12. Cassidy SB, Schwartz S, Miller JL, Driscoll DJ (2011) Prader-Willi syndrome. Genet Med.

13. Lalande M, Calciano MA (2007) Molecular epigenetics of Angelman syndrome. Cell Mol Life Sci 64: 947-960.

14. Greer PL, Hanayama R, Bloodgood BL, Mardinly AR, Lipton DM, et al. (2010) The Angelman Syndrome protein Ube3A regulates synapse development by ubiquitinating arc. Cell 140: 704-716.

15. Mishra A, Godavarthi SK, Jana NR (2009) UBE3A/E6-AP regulates cell proliferation by promoting proteasomal degradation of p27. Neurobiol Dis $36: 26-34$.

16. Chamberlain SJ (2013) RNAs of the human chromosome 15q11-q13 imprinted region. Wiley Interdiscip Rev RNA 4: 155-166.

17. Sahoo T, del Gaudio D, German JR, Shinawi M, Peters SU, et al. (2008) Prader-Willi phenotype caused by paternal deficiency for the HBII- 85 C/D box small nucleolar RNA cluster. Nat Genet 40: 719-721.

18. Chamberlain SJ, Chen PF, Ng KY, Bourgois-Rocha F, Lemtiri-Chlieh F, et al. (2010) Induced pluripotent stem cell models of the genomic imprinting disorders Angelman and Prader-Willi syndromes. Proc Natl Acad Sci U S A 107: 17668-17673.

19. Takahashi K, Yamanaka S (2006) Induction of pluripotent stem cells from mouse embryonic and adult fibroblast cultures by defined factors. Cell 126: 663-676.

20. Park IH, Arora N, Huo H, Maherali N, Ahfeldt T, et al. (2008) Diseasespecific induced pluripotent stem cells. Cell 134: 877-886.

21. Zhang N, An MC, Montoro D, Ellerby LM (2010) Characterization of Human Huntington's Disease Cell Model from Induced Pluripotent Stem Cells. PLoS Curr 2: RRN1193. 
22. An MC, Zhang N, Scott G, Montoro D, Wittkop T, et al. (2012) Genetic correction of Huntington's disease phenotypes in induced pluripotent stem cells. Cell Stem Cell 11: 253-263.

23. Song H, Chung SK, Xu Y (2010) Modeling disease in human ESCs using an efficient BAC-based homologous recombination system. Cell Stem Cell 6: 80-89.

24. Mabb AM, Judson MC, Zylka MJ, Philpot BD (2011) Angelman syndrome: insights into genomic imprinting and neurodevelopmental phenotypes. Trends Neurosci 34: 293-303.

25. Huang HS, Burns AJ, Nonneman RJ, Baker LK, Riddick NV, et al. (2013) Behavioral deficits in an Angelman syndrome model: effects of genetic background and age. Behav Brain Res 243: 79-90.

26. Gabriel JM, Merchant M, Ohta T, Ji Y, Caldwell RG, et al. (1999) A transgene insertion creating a heritable chromosome deletion mouse model of Prader-Willi and angelman syndromes. Proc Natl Acad Sci U S A 96: 9258-9263.

27. Segal DJ (2013) Bacteria herald a new era of gene editing. Elife 2: e00563.

28. Wiedenheft B, Sternberg SH, Doudna JA (2012) RNA-guided genetic silencing systems in bacteria and archaea. Nature 482: 331-338.

29. Bassett AR, Tibbit C, Ponting CP, Liu JL (2013) Highly efficient targeted mutagenesis of Drosophila with the CRISPR/Cas9 system. Cell Rep 4: 220-228.

30. Segal DJ, Meckler JF (2013) Genome engineering at the dawn of the golden age. Annu Rev Genomics Hum Genet 14: 135-158.

31. Wu X, Scott DA, Kriz AJ, Chiu AC, Hsu PD, et al. (2014) Genome-wide binding of the CRISPR endonuclease Cas9 in mammalian cells. Nat Biotechnol 32: 670-676.

32. Kuscu C, Arslan S, Singh R, Thorpe J, Adli M (2014) Genome-wide analysis reveals characteristics of off-target sites bound by the Cas 9 endonuclease. Nat Biotechnol 32: 677-683.

33. Wang H, Yang H, Shivalila CS, Dawlaty MM, Cheng AW, et al. (2013) One-step generation of mice carrying mutations in multiple genes by CRISPR/Cas-mediated genome engineering. Cell 153: 910-918.

34. Zhou J, Shen B, Zhang W, Wang J, Yang J, et al. (2014) One-step generation of different immunodeficient mice with multiple gene modifications by CRISPR/Cas9 mediated genome engineering. Int J Biochem Cell Biol 46: 49-55.

35. Ye L, Wang J, Beyer AI, Teque F, Cradick TJ, et al. (2014) Seamless modification of wild-type induced pluripotent stem cells to the natural CCR5ÎI" 32 mutation confers resistance to HIV infection. Proc Natl Acad Sci U S A 111: 9591-9596.

36. Mali P, Yang L, Esvelt KM, Aach J, Guell M, et al. (2013) RNA-guided human genome engineering via Cas9. Science 339: 823-826.

37. Cornu TI, Thibodeau-Beganny S, Guhl E, Alwin S, Eichtinger M, et al. (2008) DNA-binding specificity is a major determinant of the activity and toxicity of zinc-finger nucleases. Mol Ther 16: 352-358.

38. Pattanayak V, Ramirez CL, Joung JK, Liu DR (2011) Revealing off-target cleavage specificities of zinc-finger nucleases by in vitro selection. Nat Methods 8: 765-770.

39. Guilinger JP, Pattanayak V, Reyon D, Tsai SQ, Sander JD, et al. (2014) Broad specificity profiling of TALENs results in engineered nucleases with improved DNA-cleavage specificity. Nat Methods 11: 429-435.

40. Mussolino C, Morbitzer R, Lütge F, Dannemann N, Lahaye T, et al. (2011) A novel TALE nuclease scaffold enables high genome editing activity in combination with low toxicity. Nucleic Acids Res 39: 9283-9293.

41. Reyon D, Tsai SQ, Khayter C, Foden JA, Sander JD, et al. (2012) FLASH assembly of TALENs for high-throughput genome editing. Nat Biotechnol 30: 460-465.

42. Holkers M, Maggio I, Liu J, Janssen JM, Miselli F, et al. (2013) Differential integrity of TALE nuclease genes following adenoviral and lentiviral vector gene transfer into human cells. Nucleic Acids Res 41: e63.
43. Zhang F, Cong L, Lodato S, Kosuri S, Church GM, et al. (2011) Efficient construction of sequence-specific TAL effectors for modulating mammalian transcription. Nat Biotechnol 29: 149-153.

44. Kabadi AM, Hilton IB, Gersbach CA (2014) Multiplex CRISPR/Cas9basedgenome engineering from a single lentiviral vector. Nucleic Acids Research

45. Wang T, Wei JJ, Sabatini DM, Lander ES (2014) Genetic screens in human cells using the CRISPR-Cas9 system. Science 343: 80-84.

46. Dindot SV, Antalffy BA, Bhattacharjee MB, Beaudet AL (2008) The Angelman syndrome ubiquitin ligase localizes to the synapse and nucleus, and maternal deficiency results in abnormal dendritic spine morphology. Hum Mol Genet 17: 111-118.

47. An MC, O'Brien RN, Zhang N, Patra BN, De La Cruz M, et al. (2014) Polyglutamine Disease Modeling: Epitope Based Screen for Homologous Recombination using CRISPR/Cas9 System. PLoS Curr 6.

48. Horii T, Tamura D, Morita S, Kimura M, Hatada I (2013) Generation of an ICF syndrome model by efficient genome editing of human induced pluripotent stem cells using the CRISPR system. Int J Mol Sci 14: 19774-19781.

49. Xiao A, Wang Z, Hu Y, Wu Y, Luo Z, et al. (2013) Chromosomal deletions and inversions mediated by TALENs and CRISPR/Cas in zebrafish. Nucleic Acids Res 41: e141.

50. Yin H, W. Xue W, S. Chen S, R.L. Bogorad RL, Benedetti E et al. (2014) Genome editing with Cas9 in adult mice corrects a disease mutation and phenotype. Nat Biotechnol 32: 551-553.

51. Smith C, Gore A, Yan W, Abalde-Atristain L4, Li Z, et al. (2014) Wholegenome sequencing analysis reveals high specificity of CRISPR/Cas9 and TALEN-based genome editing in human iPSCs. Cell Stem Cell 15: 12-13.

52. Hwang WY, Fu Y, Reyon D, Maeder ML, Tsai SQ, et al. (2013) Efficient genome editing in zebrafish using a CRISPR-Cas system. Nat Biotechnol 31: 227-229.

53. Niu Y, Shen B, Cui Y, Chen Y, Wang J, et al. (2014) Generation of genemodified cynomolgus monkey via Cas9/RNA-mediated gene targeting in one-cell embryos. Cell 156: 836-843.

54. Glessner JT, Wang K, Cai G, Korvatska O, Kim CE, et al. (2009) Autism genome-wide copy number variation reveals ubiquitin and neuronal genes. Nature 459: 569-573.

55. Toro R, Konyukh M, Delorme R, Leblond C, Chaste P, et al. (2010) Key role for gene dosage and synaptic homeostasis in autism spectrum disorders. Trends Genet 26: 363-372.

56. Ghahramani Seno MM, Hu P, Gwadry FG, Pinto D, Marshall CR, et al. (2011) Gene and miRNA expression profiles in autism spectrum disorders. Brain Res 1380: 85-97.

57. Fu Y, Sander JD, Reyon D, Cascio VM, Joung JK (2014) Improving CRISPR-Cas nuclease specificity using truncated guide RNAs. Nat Biotechnol 32: 279-284.

58. Mali P, Aach J, Stranges PB, Esvelt KM, Moosburner M, et al. (2013) CAS9 transcriptional activators for target specificity screening and paired nickases for cooperative genome engineering. Nat Biotechnol 31: 833-838.

59. Ran FA, Hsu PD, Lin CY, Gootenberg JS, Konermann S, et al. (2013) Double nicking by RNA-guided CRISPR Cas9 for enhanced genome editing specificity. Cell 154: 1380-1389.

60. Tsai SQ, Wyvekens N, Khayter C, Foden JA, Thapar V, et al. (2014) Dimeric CRISPR RNA-guided FokI nucleases for highly specific genome editing. Nat Biotechnol 32: 569-576.

61. Huang HS, Allen JA, Mabb AM, King IF, Miriyala J, et al. (2011) Topoisomerase inhibitors unsilence the dormant allele of Ube3a in neurons. Nature 481: 185-189.

62. King IF, Yandava CN, Mabb AM, Hsiao JS, Huang HS, et al. (2013) Topoisomerases facilitate transcription of long genes linked to autism. Nature 501: 58-62.

63. Shalem O, Sanjana NE, Hartenian E, Shi X, Scott DA, et al. (2014) Genome-scale CRISPR-Cas9 knockout screening in human cells. Science 343: 84-87. 